\title{
Gridless Determination of Aerodynamic Loads Using Lagrangian Particle Tracks
}

\author{
Christoph Mertens*, José L. Costa Fernández, Andrea Sciacchitano, \\ Bas W. van Oudheusden, Jurij Sodja
}

Delft University of Technology, Faculty of Aerospace Engineering, 2629HS Delft, The Netherlands

*C.Mertens@tudelft.nl

\begin{abstract}
The aerodynamic loads on a flexible wing in terms of the surface pressure distribution and the lift force along the span are determined experimentally based on non-intrusive Lagrangian particle tracking (LPT) measurements. As the flexible wing deforms under the aerodynamic loads, its deformed shape is first reconstructed based on structural LPT measurements conducted together with the flow measurements in an integrated approach. Based on the reconstructed wing shape, flow tracers data are collected along surface normals to evaluate the surface pressure, as well as along elliptic paths around the wing to determine the circulation. The lift force is calculated from the surface pressure by integrating the pressure difference along the chord, as well as from the circulation using the Kutta-Joukowski theorem. The circulation-based lift results are in very good agreement with reference measurements from a force balance, with differences in the total lift force on the wing of less than 5\%. The lift estimation based on the extrapolated surface pressure is consistently lower than the circulation-based lift, by about $10 \%$, due to the limited accuracy of the pressure extrapolation near the leading edge region, where a considerable fraction of the lift is generated.
\end{abstract}

\section{Introduction}

The experimental determination of the aerodynamic loads that act on flexible wings is relevant for aeroelastic research and development activities, in particular for providing reference data to validate numerical aeroelastic models. The situation of aerodynamic loads on highly flexible wings is particularly relevant because these wings can exhibit nonlinear structural behavior, which increases the complexity of the numerical aeroelastic model and as such the need for experimental validation data (Dimitriadis (2017)).

The installation of pressure sensors to measure the aerodynamic load on such highly flexible wings is difficult, or, depending on the respective design of the flexible wing skin, may even be impossible. It is therefore preferred to determine the aerodynamic loads non-intrusively, which can be achieved by measuring the flow field around the wing and then inferring the aerodynamic loads from these measurements by using the governing equations of the flow (van Oudheusden (2013); Rival and van Oudheusden (2017)).

Lagrangian particle tracking (LPT) via the Shake-The-Box algorithm (Schanz et al. (2016)) in combination with the use of helium-filled soap bubbles (HFSB) as flow tracers (Scarano et al. (2015)) facilitates the performance of volumetric flow field measurements at a relatively large scale, which is required to obtain the aerodynamic loads in the relevant aeroelastic regimes. A further advantage of this technique is that it facilitates the simultaneous characterization of the structural response and the three-dimensional flow topology with an integrated approach, as described in Mertens et al.(2021).

However, a drawback of the LPT flow field measurements is its relatively poor spatial resolution, caused by the relatively large inter-particle distance, especially when large-scale measurements with HFSB are conducted. A method to mitigate this limitation is to perform a statistical analysis of the LPT data throughout the flow field by ensemble-averaging the track data in time on a Cartesian grid (Agüera et al. (2016)). A considerable disadvantage of this procedure for the application in a loads determination procedure on flexible wings is that the grid of the flow field does not automatically comply with the deformed shape of the wing, which impedes the loads determination.

In this study, it is demonstrated how the particle track data from the LPT measurements of the flow field and the structure can be used to determine the aerodynamic loads on a flexible wing without requiring the 
additional step of ensemble averaging the LPT flow measurements on a regular grid. An important first step in this approach is the determination of the deformed shape of the wing under the aerodynamic load, which is performed based on the LPT measurements of structural markers. Subsequently, two different approaches are considered to determine the aerodynamic load from the flow measurements. In the pressurebased approach, the measured shape of the wing is used to collect LPT data in the flow field along surface normals to find the pressure on the wing surface by extrapolation. In the circulation-based approach, the flow field LPT data is collected on elliptic contours around the wing to determine the lift force across the span. The loads are calculated using relations from potential flow theory, which only require measurements of the velocity, that are directly available from the individual LPT data points. Results are compared to reference measurements of the overall lift force across the span from a force balance.

\section{Experimental Procedures}

\subsection{Wind Tunnel Setup}

The experiments were conducted in the Open Jet Facility at Delft University of Technology, which is an open test section, closed return wind tunnel with an octagonal outlet of $2.85 \mathrm{~m} \times 2.85 \mathrm{~m}$. The test object was a highly flexible wing that is similar to the aeroelastic benchmark model design that is described in Avin et al. (2021), which can sustain very large deformations, with wingtip displacements of up to $50 \%$ of its span width. The wind tunnel was operated at a freestream velocity of $U_{\infty}=18.3 \mathrm{~m} / \mathrm{s}$, corresponding to a Reynolds number of $\operatorname{Re}=122000$ based on the wing chord. Optical measurements of the flow and the position of the wing were conducted simultaneously with an integrated measurement approach. A sketch of the flexible wing and a photo of the measurement setup in the wind tunnel are shown in Fig. 1.

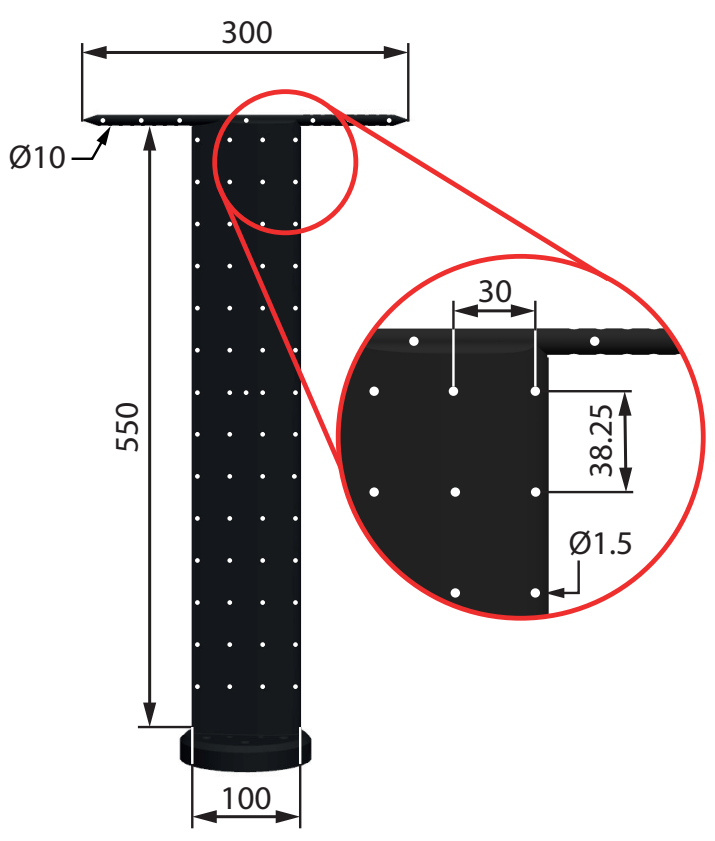

(a)

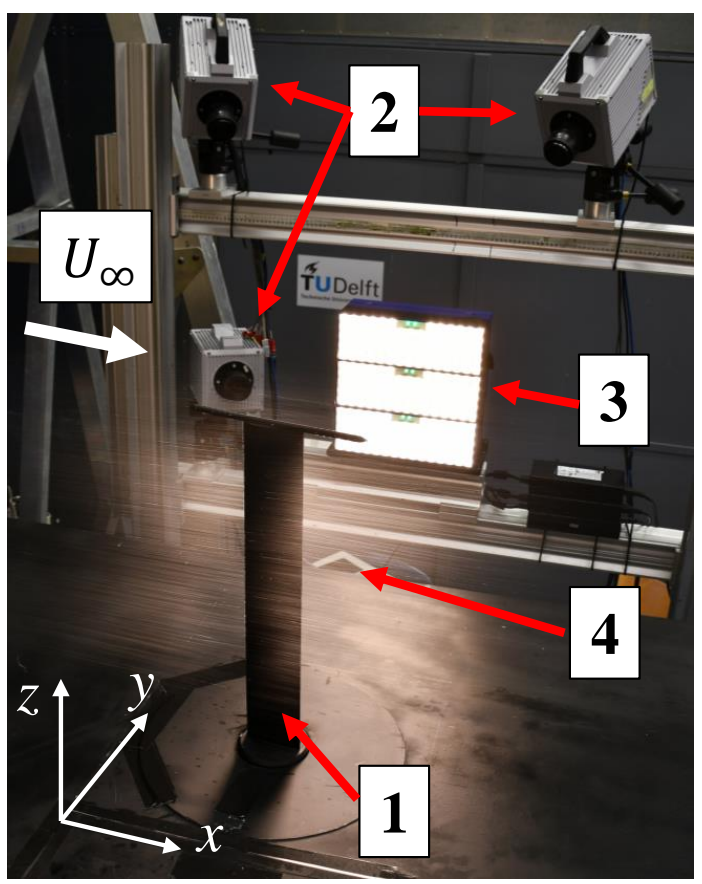

(b)

Figure 1: Experimental model and wind tunnel setup, (a) sketch with dimensions (in millimeter) of the flexible wing and structural markers grid, (b) photo of the experimental setup, 1: flexible wing, 2: highspeed cameras, 3: LED illumination units, 4: stream of helium-filled soap bubbles

The flexible wing model that is illustrated in Fig. 1(a) has a chord length of $c=100 \mathrm{~mm}$ and a span width of $s=550 \mathrm{~mm}$ with a NACA 0018 airfoil section. The wing structure is a 3D printed Nylon 12 chassis, with a mounting base and a $300 \mathrm{~mm}$ long tip rod, and an Aluminum 7075 spar with a thickness of $1.5 \mathrm{~mm}$ that is bonded to the chassis using Loctite 495 glue. The wing skin is made out of black Oralight polyester foil. 
Structural measurements of the wing were performed by tracking reflective markers on the surface. For this purpose, white circular markers with a diameter of $1.5 \mathrm{~mm}$ were painted on the wing surface using a laser-cut mask, markers were painted on the tip rod and at the spanwise locations of the ribs, which have a spacing of $38.25 \mathrm{~mm}$, with a chordwise spacing of the markers of $30 \mathrm{~mm}$.

As visible in Fig. 1(b), the wing was mounted vertically in the wind tunnel test section. It was mounted on a six-component force balance that was connected to a rotating table, which was used to set the geometric angle of attack $\alpha$ of the wing. In this study, two different angles are investigated, $\alpha_{1}=5^{\circ}$ and $\alpha_{2}=10^{\circ}$, and force measurements acquired with the balance are used as a reference for the aerodynamic loads that are determined based on the optical LPT measurements.

The optical measurement system that is shown in Fig. 1(b) consists of three Photron Fastcam SA1.1 high-speed cameras with a sensor size of $1024 \times 1024$ pixels and an image sampling rate of $5.4 \mathrm{kHz}$. The flow measurements were performed using helium-filled soap bubbles (HFSB) as tracer particles (Scarano et al. (2015)). The HFSB were produced with a seeding generator that is placed in the settling chamber of the wind tunnel, upstream of the contraction of the wind tunnel nozzle. The seeding generator consists of 200 bubble-producing nozzles distributed over an area of $500 \mathrm{~mm} \times 1000 \mathrm{~mm}$. The working principle of the bubble-producing nozzles is described in Faleiros et al. (2019). The resulting HFSB seeding concentration was around $1 \mathrm{~cm}^{-3}$. The HFSB flow tracers were illuminated with three LaVision LED-Flashlight 300 modules, and the image acquisition and processing were performed with the LaVision Davis 10 software.

\subsection{Data Acquisition and Processing}

The data acquisition and processing to obtain the LPT measurements of the flow and the structure closely follows the procedure of the integrated measurement approach described in Mertens et al. (2021). This procedure begins with the optical calibration of the system by performing a volume self-calibration (Wieneke (2008)) and generating an optical transfer function (Schanz et al.(2013)). Afterward, the integrated flow and structural measurements are separated, as illustrated in Fig. 2. Optical measurements of the flow tracers and the structural markers are acquired by the cameras, as shown in Fig. 2(a), To obtain the isolated information of only the flow or the structure, temporal filters are applied to the time series of the images; the application of a temporal high pass filter produces the isolated flow tracers information (Sciacchitano and Scarano (2014), see Fig. 2(b)), and a temporal low-pass filter produces the isolated structural information (Mitrotta et al., see Fig. 2(c)). Afterward, the LPT measurements of the structure and the flow are generated by applying the Shake-The-Box algorithm (Schanz et al. (2016)) to the separate image data sets.

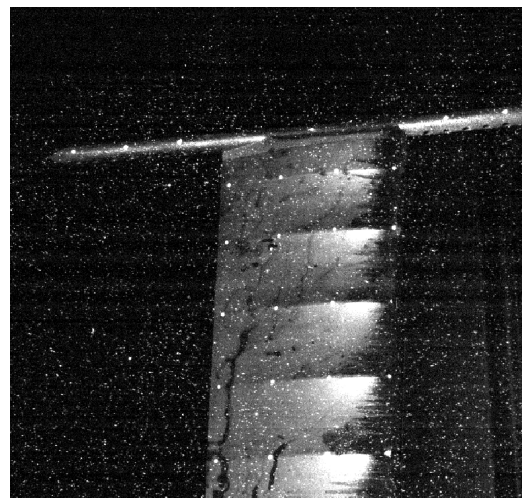

(a)

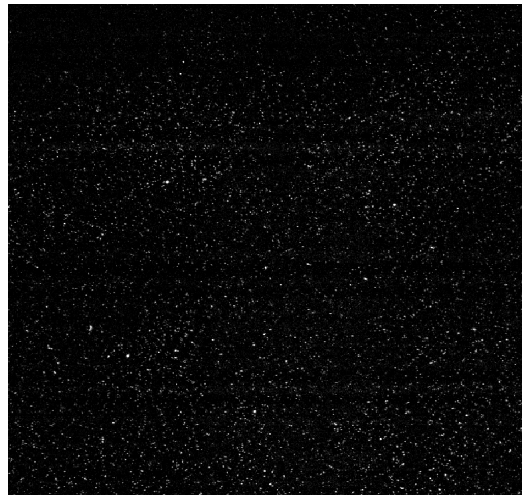

(b)

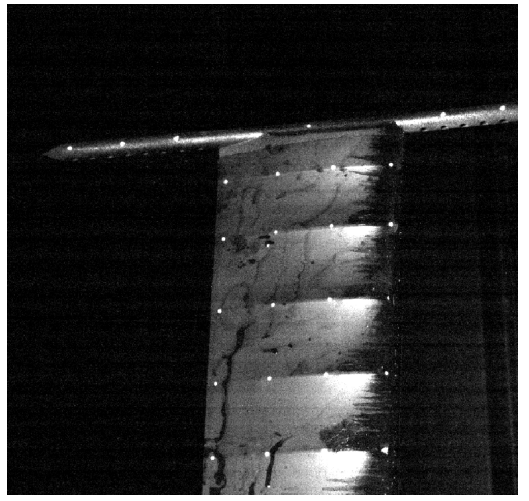

(c)

Figure 2: Image data processing procedure: (a) integrated optical measurement of flow and structure, (b) image data of the flow tracers, (c) image data of the structure

The size of the measurement volume that was achieved with the optical measurement setup was around 27 liters $(300 \mathrm{~mm} \times 300 \mathrm{~mm} \times 300 \mathrm{~mm})$, although part of this volume was obstructed by the presence of the wing, such that the LPT measurements were obtained within a volume of around 22 liters. For the aerodynamic loads determination, measurements from both sides of the wing and along the entire span are necessary. To obtain this data with the described measurement setup, the LPT measurements were performed in four separate acquisitions. First, two acquisitions were performed for the bottom half of the wingspan, where the angle of attack of the wing was set to $+\alpha$ and $-\alpha$, respectively. The data acquired at 
$-\alpha$ (pressure side) was later mirrored in post-processing to the opposite side of the wing and merged with the data acquired at $+\alpha$ (suction side). Thereby, the symmetry of the wing was exploited to simplify the LPT data acquisition, because, with this approach, it is not necessary to move and recalibrate the optical measurement setup. The same procedure is repeated for the top half of the wingspan.

The resulting four separate acquisitions are merged by transforming the LPT data from the measurement coordinate system to the wind tunnel coordinate system with a rigid-body transformation. The translation and rotation matrices for this rigid-body transformation are determined based on reference LPT measurements of the structural markers on the wing, performed without wind tunnel operation before each manipulation of the measurement volume. The accuracy of the employed merging procedure can be assessed by comparing the flow velocity at corresponding positions with respect to the wing from different acquisitions. Typical values of these differences were between $1 \%$ and $3 \%$ of the local velocity magnitude. Since these values are of the same order of magnitude as when the merging of different flow measurement acquisitions is performed automatically based on position measurements with a robotic arm as in Jux et al. (2018), these differences are considered acceptable and not further investigated.

\section{Wing Shape Reconstruction}

After the LPT measurements of the structural markers are transformed from the measurement coordinate system to the wind tunnel coordinate system, the marker position measurements are used to reconstruct the deformed shape of the wing. This is achieved by calculating the wing deflection as an average value of the marker positions for each spanwise section where the markers were painted (i.e., the ribs of the wing and the tip rod) and then fitting a polynomial through these measurements along the spanwise direction. Following this procedure, the result of the polynomial curve fit is used as a reference spanwise axis to calculate the deformed wing shape. A fourth-order polynomial is used in this study to perform the curve fit, which satisfies the geometric boundary conditions of a wing that is clamped at the root, i.e. $d(z=0)=0$ and $d^{\prime}(z=0)=0$, and is defined as

$$
d(z)=A z^{4}+B z^{3}+C z^{2}
$$

where the coefficients $A, B$, and $C$ are determined with an optimization to provide the best fit to the experimental measurements in a least-squared sense. The individual marker measurements from 100 acquired images are averaged to reduce the effect of measurement noise and small-scale vibrations of the wing during the experiment. The results of the polynomial curve fit to the marker measurements for the two different values of $\alpha$ are shown in Fig. 3. The standard deviation of the residual between the 15 measurement locations along the span and the curve fit is $\sigma_{1}=0.13 \mathrm{~mm}$ for $\alpha_{1}$ and $\sigma_{2}=0.25 \mathrm{~mm}$ for $\alpha_{2}$, corresponding to $0.28 \%$ and $0.29 \%$ of the respective wingtip displacement. Note that the value of the spanwise position of the wingtip can exceed $z_{\text {tip }} / s=1$ because there are markers on the wingtip rod, which is not accounted for in the definition of the span length $s$.

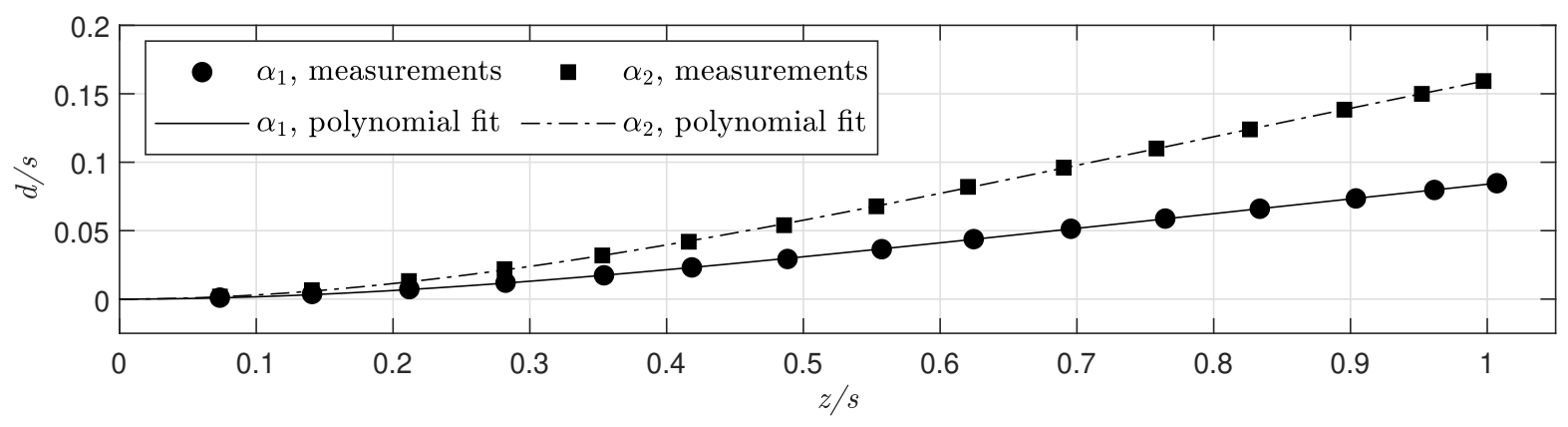

Figure 3: Displacement measurements along the span with polynomial curve fit

To further characterize the deformed wing shape, knowledge about the torsional deformation is required as well. The torsional deformation can be expressed in terms of a twist angle $\varepsilon$ of the wing around the reference axis. The twist angle $\varepsilon$ of the wingtip can be estimated from the structural marker measurements on the tip rod. The experimentally determined twist angles were found to be $\varepsilon_{1}=0.26^{\circ}$ for $\alpha_{1}$ and $\varepsilon_{2}=0.45^{\circ}$ for $\alpha_{2}$. Based on these small values of $\varepsilon$ and to simplify the analysis, the twist has been neglected in the further analysis, assuming that the deformed wing shape can be reconstructed with sufficient accuracy directly from the polynomial curve fit of the deflection along the span. 


\section{Loads Determination Methods}

\subsection{Surface Pressure Determination}

In incompressible, inviscid, and irrotational steady flow, the local flow velocity and pressure in the entire field are related through Bernoulli's equation, yielding

$$
C_{p}=\frac{p-p_{\infty}}{\frac{1}{2} \rho U_{\infty}^{2}}=1-\frac{V^{2}}{U_{\infty}^{2}},
$$

where $p$ is the pressure, $\rho$ is the fluid density, and $V$ is the velocity magnitude. For attached flow around wings, the aforementioned conditions for the application of Bernoulli's equation are generally justifiable assumptions, except for the viscous flow inside the boundary layer. The presence of the boundary layer and, in view of random measurement errors, the prevalence of relatively strong velocity gradients near the surface obstruct a direct application of Eq. 2 to the LPT measurements in the vicinity of the surface to determine the local surface pressure. It is therefore necessary to perform an extrapolation of the pressure obtained with Eq. 2 from the flow field to the surface, as demonstrated in Mertens et al. (2021).

The LPT flow measurements provide discrete values for the pressure in the flow field by applying Eq. 2 to each particle track. For the surface pressure extrapolation in this study, the LPT measurements within a given radius around a finite number of surface normals from the wing are used, instead of first interpolating them onto a regular grid. The surface normals are computed from the reconstructed wing shape by discretizing the wing shape with planar rectangular elements and then calculating the normal direction $n$ and the tangential direction $t$ from a sampling point at the center of each element. The coordinates of the LPT measurements are then transformed into the local coordinate system of the wing so that the data within a given normal and tangential distance around each surface normal can be used for the extrapolation of the surface pressure at the respective sampling point on the wing. The data collection procedure is illustrated schematically in Fig. 4 .

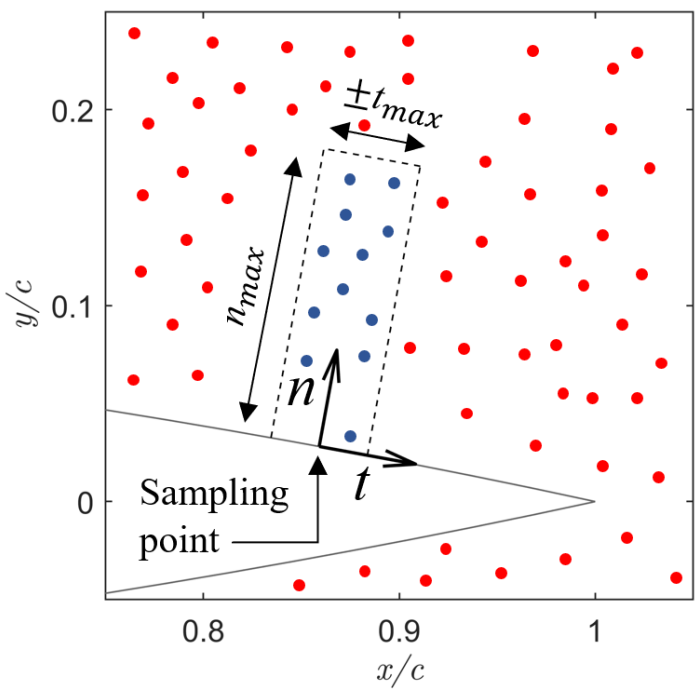

Figure 4: Sketch of the LPT data collection procedure for a sampling point at $x / c=0.86$. Blue dots: LPT track data considered for the pressure extrapolation, red dots: LPT track data that is not considered

In Fig. 5, the LPT measurements along 100 surface normals with a length of $n_{\max }=15 \mathrm{~mm}(0.15 c)$ are shown for a cross-section of the wing at $\alpha_{2}=10^{\circ}$ for the spanwise location $z / s=0.6$. There are 50 normals on the suction (top) and pressure (bottom) side, respectively. The sampling points on the surface are distributed using a half-cosine spacing along the chord to concentrate the sampling points in the leading edge region of the airfoil where high pressure gradients are expected. The LPT data within a spanwise region that extends over $11 \mathrm{~mm}( \pm 0.01 s)$ around the spanwise location $z / s=0.6$ is considered. Within this spanwise region, the LPT data with a maximum tangential distance of $t_{\max }=2.5 \mathrm{~mm}(0.025 c)$ to the respective surface normal is collected and plotted along the normal in Fig. 5 as a function of its respective wall distance $n$. 


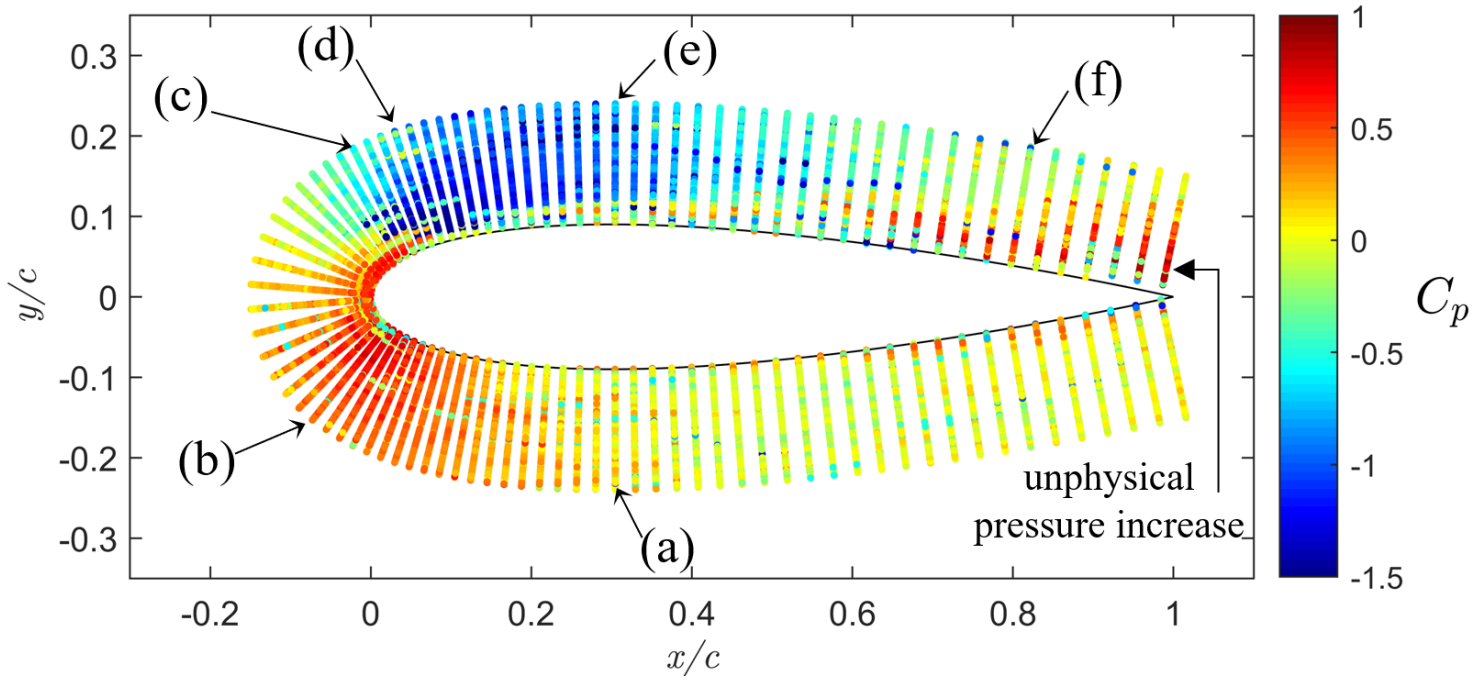

Figure 5: LPT-based pressure from Bernoulli's equation along surface normals at $z / s=0.6$ for $\alpha_{2}=10^{\circ}$. The labels (a-f) in the figure indicate the normals that are plotted in Fig. 6

As visible in Fig. 5, a considerable amount of random measurement noise and artifacts obstruct the application of Eq. 2 in the direct vicinity of the surface. In particular, an unphysical increase in the pressure obtained with Eq. 2 is observed close to the wing surface, most prominently near the trailing edge on the suction side. In this region, the presence of the boundary layer decelerates the flow near the wing surface, which according to Eq. 2 implies an increase in pressure, whereas it is known from boundary layer theory that the pressure does not vary across the boundary layer (see Schlichting and Gersten (2017)). To determine the surface pressure from the pressure in the flow field as obtained from the LPT measurements with Eq. 2 despite the presence of the boundary layer and the random measurement errors, an extrapolation of the surface pressure is performed by fitting a polynomial through the LPT data points in the wall-normal direction using a least-squares method. A second-order polynomial is used and data points with a wallnormal distance $n$ up to $n_{\max }=0.15 \mathrm{c}$ are considered for the fit. As for the illustration in Fig. 4 and the measured data plotted in Fig. 5. LPT data from a spanwise region of $\pm 0.01 \mathrm{~s}$ around the reference position, with a maximum tangential distance of $\pm t_{\max }=0.025 \mathrm{c}$ from the LPT data point to the respective surface normal in the cross-sectional plane is considered for the pressure extrapolation. The pressure extrapolation is illustrated in Fig. 6 for the six example locations at $z / s=0.6$ of which the locations are indicated in Fig. 5 .

In general, the value of the polynomial at the wall is considered as the extrapolated surface pressure. The LPT data, the fitted polynomial, and the extrapolated surface pressure are indicated for $x / c=0.3$ on the pressure side in Fig. 6(a). The pressure extrapolation works as expected, despite the presence of a considerable amount of random error. The presence of random measurement errors is more critical when the error is not normally distributed, as near the stagnation point, which is illustrated in Fig. 6(b), where all the outliers are associated with lower pressures. The effect of these outliers is diminished by using a robust least-squares polynomial fit with bisquare weights 1 , which is used at all chordwise locations. An extraordinarily challenging measurement region is the leading edge region on the suction side, which is the flow region in between the stagnation point and suction peak, where the spatial gradients of the flow velocity and the pressure are maximal. In this region, the measured pressure data near the wall exhibits a bifurcation behavior, as shown in Figs. 6(c) and 6(d), which can be expected as a consequence of the non-zero value of $t_{\max }$ and the large pressure gradients in the wall-tangential direction in this region. As a result of the robust fitting approach, the polynomial fit follows the branch with the larger amount of measured data points in Figs. 6(c) and 6(d) A different issue of the LPT-based pressure measurement estimation with Bernoulli's equation is the presence of the boundary layer where Eq. 2 incorrectly implies an increase in pressure, in particular on the suction side. The effect of this issue on the curve fit is still negligible at $x / c=0.3$ as shown in Fig. 6(e), but clearly relevant further downstream, as shown in Fig. 6(f)] Because the actual pressure does not vary across the boundary layer, the pressure outside of the boundary layer, where Bernoulli's equation is applicable, should be used for the extrapolation of the surface pressure, instead of the value of the polynomial

1 https://mathworks.com/help/curvefit/least-squares-fitting.html 
fit at the wall. In Figs. 6(e) and 6(f), it is shown that this value can be determined on the suction side by considering the minimum value of the polynomial fit in the wall-normal direction. This approach is used for all sampling points on the suction side of the wing downstream of $x / c=0.2$, as these locations are most critically affected by the presence of the boundary layer.

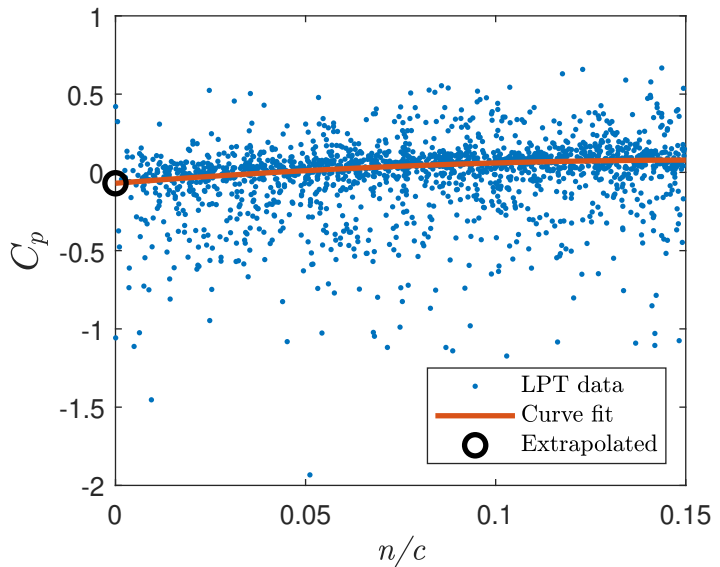

(a) $x / c=0.30$, pressure side

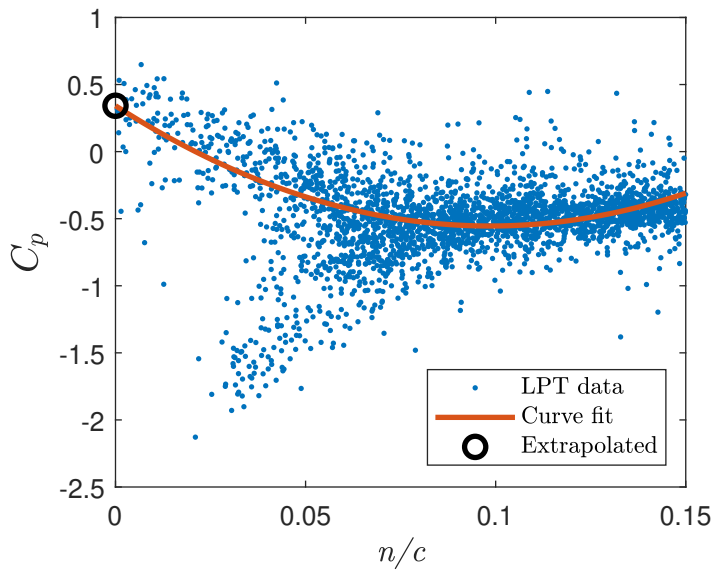

(c) $x / c=0.036$, suction side

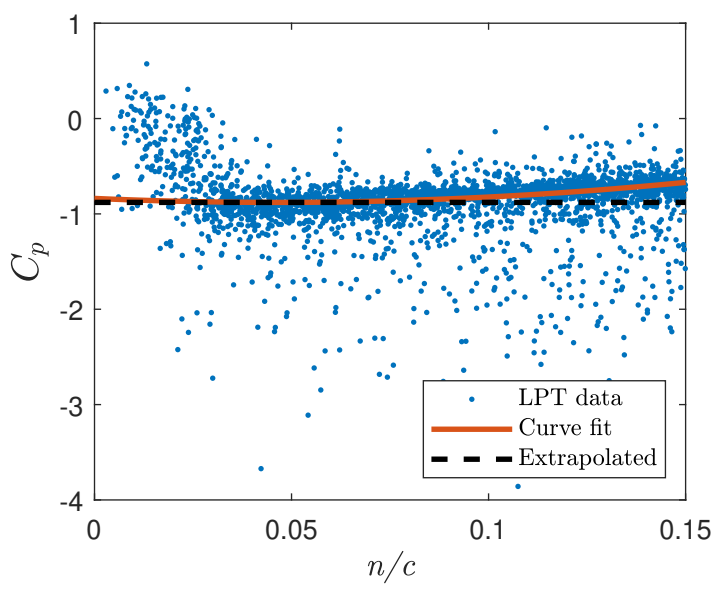

(e) $x / c=0.30$, suction side

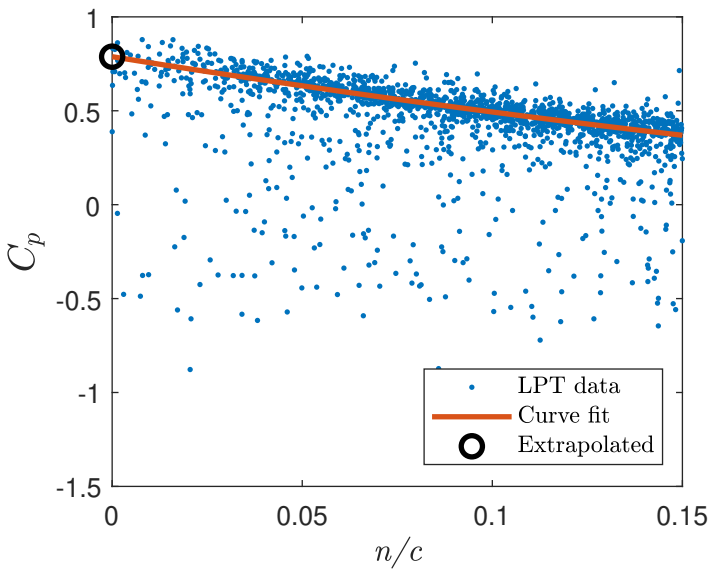

(b) $x / c=0.028$, pressure side

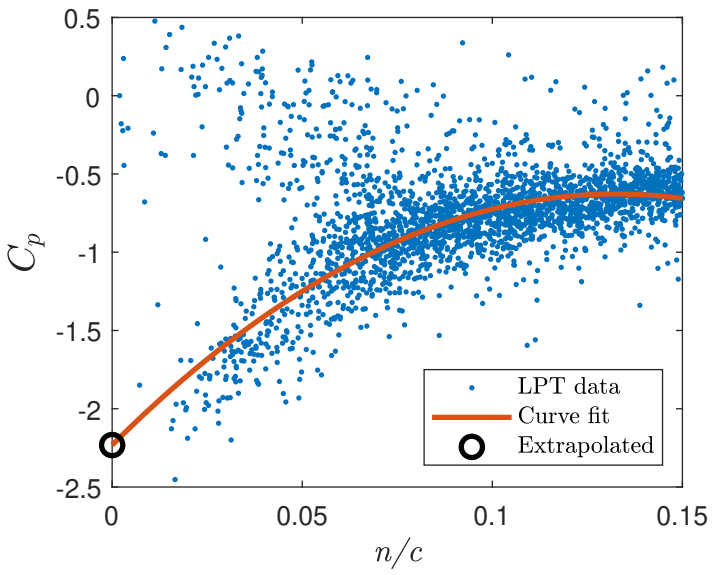

(d) $x / c=0.065$, suction side

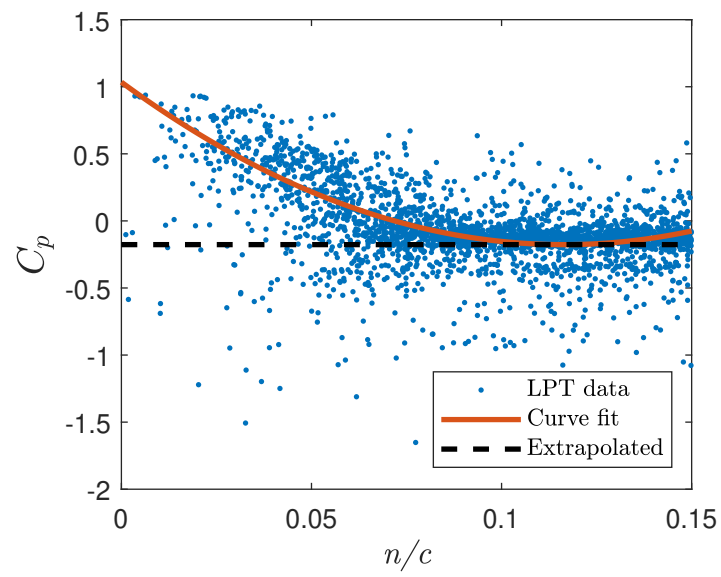

(f) $x / c=0.80$, suction side

Figure 6: Surface pressure extrapolation at six different example locations at $z / s=0.6$ for $\alpha_{2}=10^{\circ}$ 


\subsection{Lift Force Determination}

For attached flow around wings at small angles of attack $\alpha$, the lift force perpendicular to the inflow can be assumed as equivalent to the normal force on the wing, which acts perpendicular to the chord. For thin objects like airfoils, the sectional lift force can therefore be obtained by integrating the pressure difference between the suction and pressure sides along the chord, with the section lift in coefficient form given as

$$
C_{\ell}=\frac{L^{\prime}}{\frac{1}{2} \rho U_{\infty}^{2} c}=\int_{0}^{c}\left(C_{p, \text { pressure }}-C_{p, \text { suction }}\right) d x
$$

where $L^{\prime}$ is the sectional lift force per unit span, and $C_{p}$ can be determined from the flow field as described before. If the determination of the pressure distribution itself is not of primary interest, the section lift $L^{\prime}$ can alternatively be determined by using the Kutta-Joukowski theorem:

$$
L^{\prime}=\rho U_{\infty} \Gamma,
$$

where $\Gamma$ is the circulation around the wing, which can be determined by performing a line integral of the flow velocity along a closed contour $C$ around the wing:

$$
\Gamma=-\oint_{C} \vec{u} \cdot d \vec{s}
$$

Although the Kutta-Joukowski theorem is derived based on the assumption of potential flow, previous studies have shown that it can be used to determine the lift accurately from experimental data for flows that exhibit moderate effects of viscosity, and when the circulation is calculated from velocity measurements outside of the region affected by these viscous effects (Mertens et al. (2021); Sharma and Deshpande (2012); Olasek and Karczewski (2021)).

In this study, the circulation is determined from the LPT measurements by defining an elliptic path around the wing section of interest and collecting the LPT data within a prescribed distance around this path, which is then integrated along the tangential direction to determine the circulation. Because the lift force acts perpendicular to the freestream and the spanwise axis of the wing, the elliptic integration paths are defined in a local coordinate system of the respective wing section of interest, which is defined using the experimentally reconstructed wing shape. The ellipse has its origin at the mid-chord position of the wing section, the major axis is aligned with the chord and the minor axis is perpendicular to the chord in the cross-sectional plane. An example of the circulation determination procedure is visualized for $\alpha_{2}=10^{\circ}$ at $z / s=0.6$ in Fig. 7 .

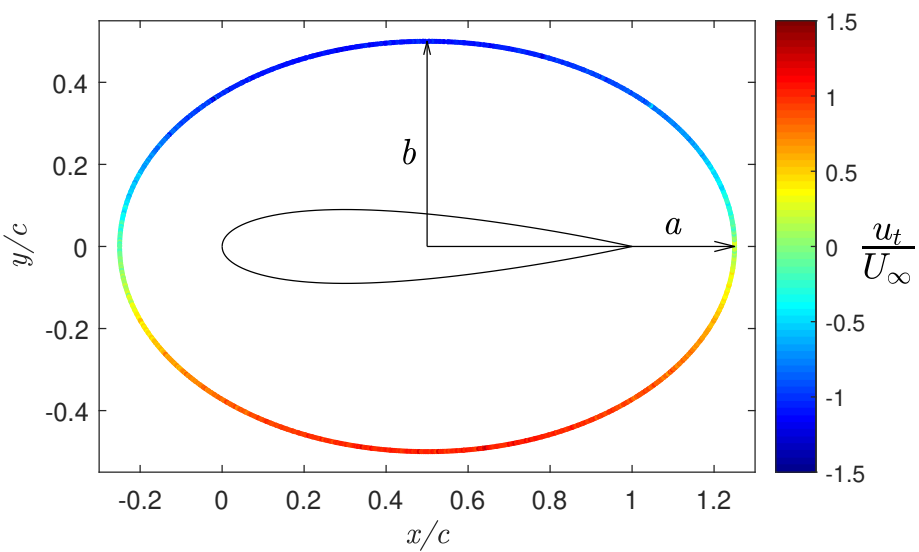

(a) LPT data on an elliptic contour, colored by tangential velocity

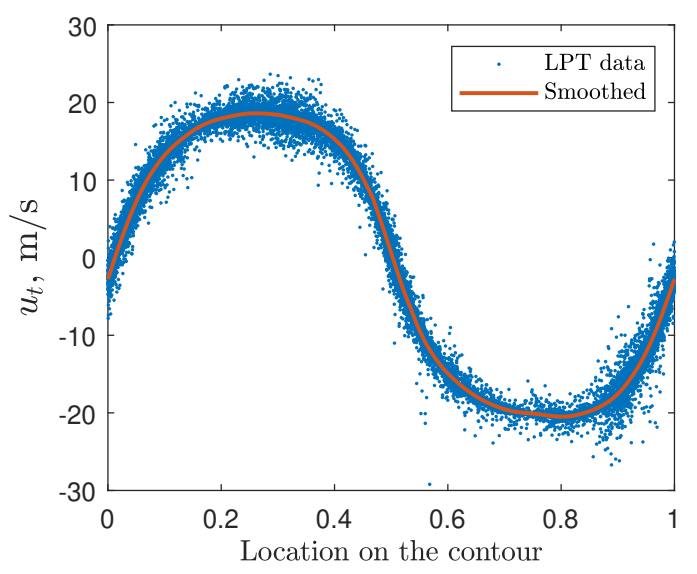

(b) Tangential velocity along the elliptic contour

Figure 7: Illustration of the circulation determination procedure at $z / s=0.6$ for $\alpha_{2}=10^{\circ}$

In Fig. 7(a), the LPT data along an elliptic contour around the wing at $z / s=0.6$ is visualized. The elliptic contour has a major semi-axis length of $a / c=0.75$ and a minor semi-axis length of $b / c=0.5$. As 
for the pressure extrapolation, LPT data within a spanwise region that extends over $11 \mathrm{~mm}( \pm 0.01 \mathrm{~s})$ around the respective spanwise location is considered. The maximum distance in the cross-sectional plane of the LPT data point to the ellipse to be considered for the circulation determination is taken as $1 \mathrm{~mm}(0.01 c)$. The tangential velocity component $u_{t}$ of the LPT data at $z / s=0.6$ along the elliptic contour is plotted in Fig. 7(b) The origin of the contour is located upstream of the wing, aligned with the chordwise direction. The positive direction along the contour is counter-clockwise around the wing. To determine the circulation from this data, the tangential velocity of the individual LPT data points is filtered with a smoothing spline ${ }^{2}$ The smoothed data is then integrated along the length of the contour to obtain the circulation.

While in the theoretical case of a potential flow the circulation would be independent of the contour as long as the object is fully enclosed by it, this assumption cannot be directly made for the present case that includes some effects of viscosity as well as due to the occurrence of measurement errors. Therefore, the effect of the ellipse geometry parameters $a$ and $b$ on the circulation $\Gamma$ is analyzed as shown in Fig. 8 for $\alpha_{1}$ and $\alpha_{2}$ at $z / s=0.6$.

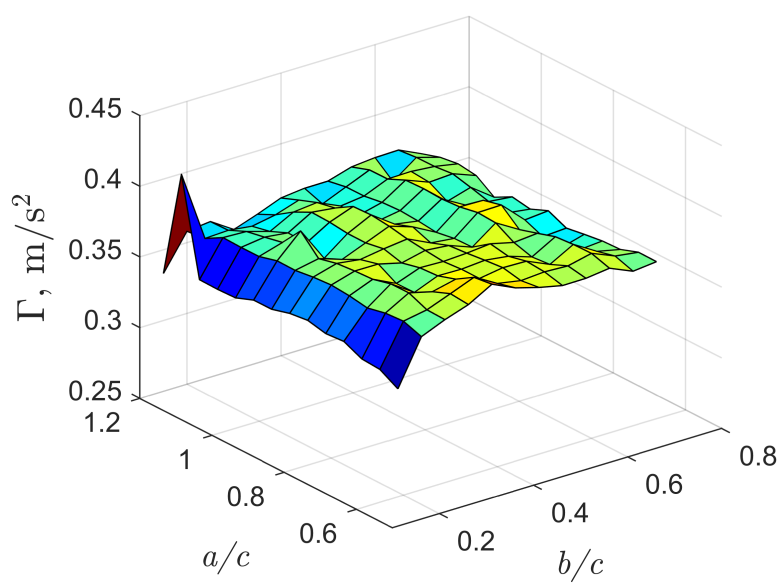

(a) $\alpha_{1}=5^{\circ}$

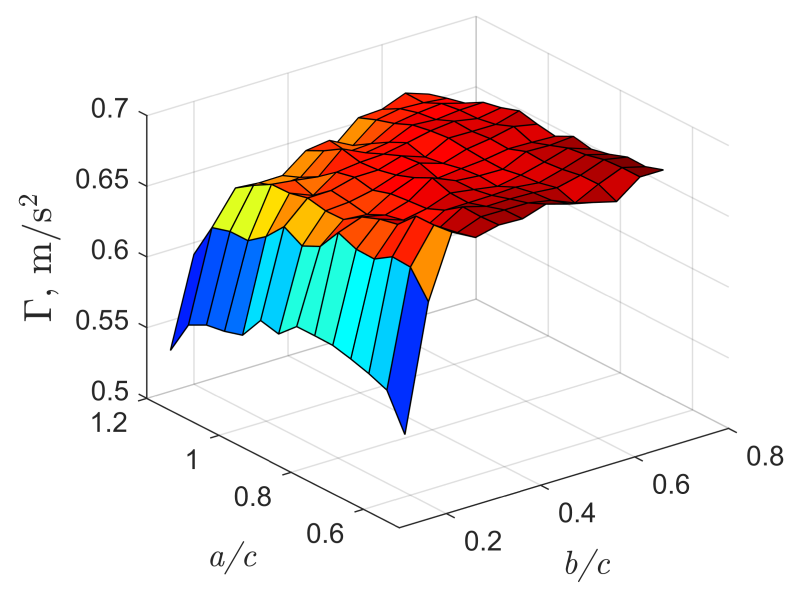

(b) $\alpha_{2}=10^{\circ}$

Figure 8: Effect of the elliptic contour parameters on the circulation at $z / s=0.6$

A relatively strong sensitivity of $\Gamma$ to $b$ is observed when the elliptic contour is in the immediate vicinity of the wing, i.e. when the contour is located within the boundary layer. This effect is amplified for $\alpha_{2}=$ $10^{\circ}$ as seen in Fig. 8(b), where the boundary layer thickness on the suction side is increased due to a stronger adverse pressure gradient, when compared to $\alpha_{1}=5^{\circ}$ in Fig. 8(a). Further away from the wing, the circulation does not vary systematically with $a$ or $b$, but it is subject to some level of variation, likely due to random measurement errors. To minimize the influence of these random errors, the circulation $\Gamma$ for a given spanwise location of the wing is determined in this study by averaging the value of $\Gamma$ from 100 different elliptic contours at that spanwise location, where the parameters of the ellipse are varied between $0.65 \leq a / c \leq 1.1$ and $0.25 \leq b / c \leq 0.7$.

\section{Results}

The results for the LPT-based pressure determination at four different spanwise locations for $\alpha_{1}$ and $\alpha_{2}$ are shown in Fig. 9. As expected, the larger angle of attack $\alpha_{2}$ produces higher pressures on the bottom and lower pressures on the top side. Within the range of $z$ shown in Fig. 9 no large variation of the pressure distributions with the spanwise position is observed. As an effect of the bifurcation in the data used for the pressure extrapolation near the leading edge that was discussed in the context of Fig. 6, the pressure coefficient $C_{p}$ is positive on the suction side in the region from the leading edge until around $x / c=0.05$ in all pressure distributions in Fig. 9 .

\footnotetext{
${ }^{2}$ https://mathworks.com/help/curvefit/smoothing-splines.html
} 


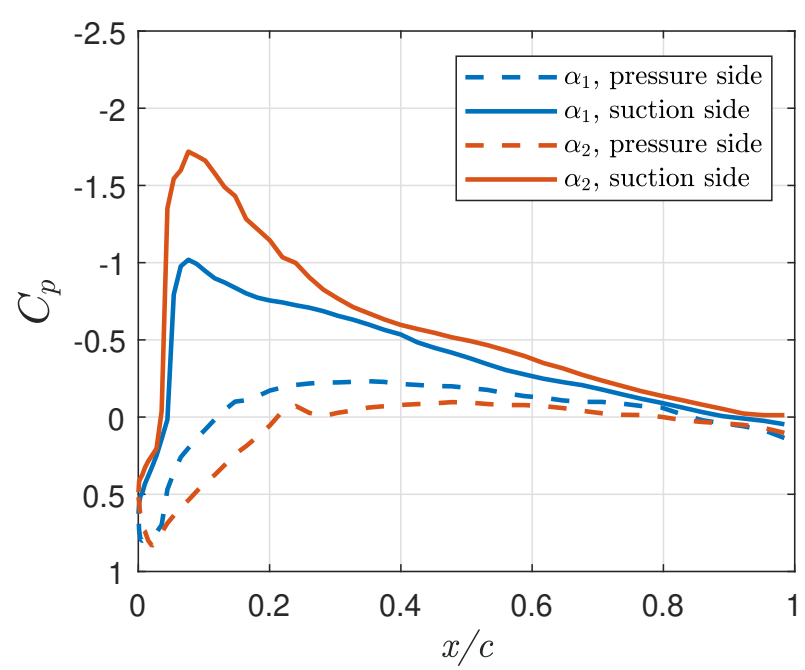

(a) $z / s=0.2$

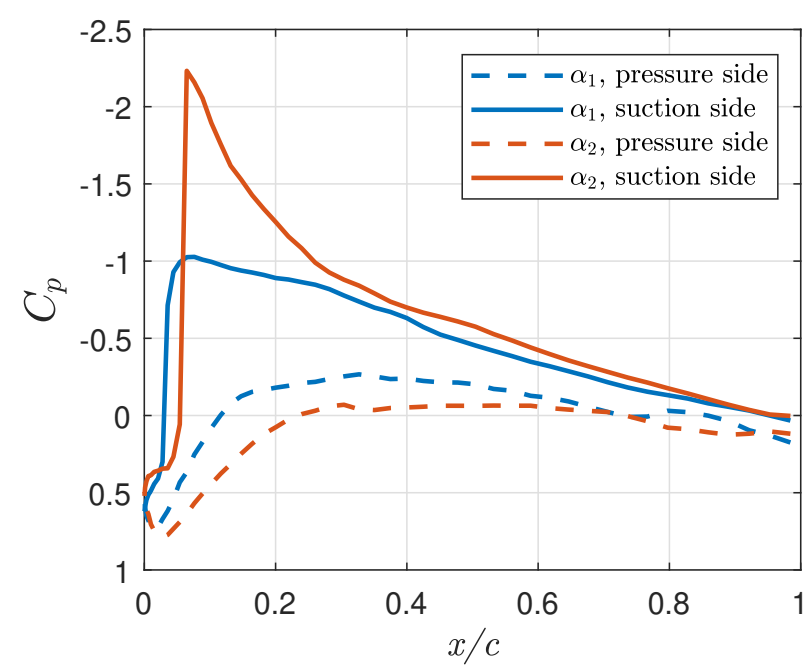

(c) $z / s=0.6$

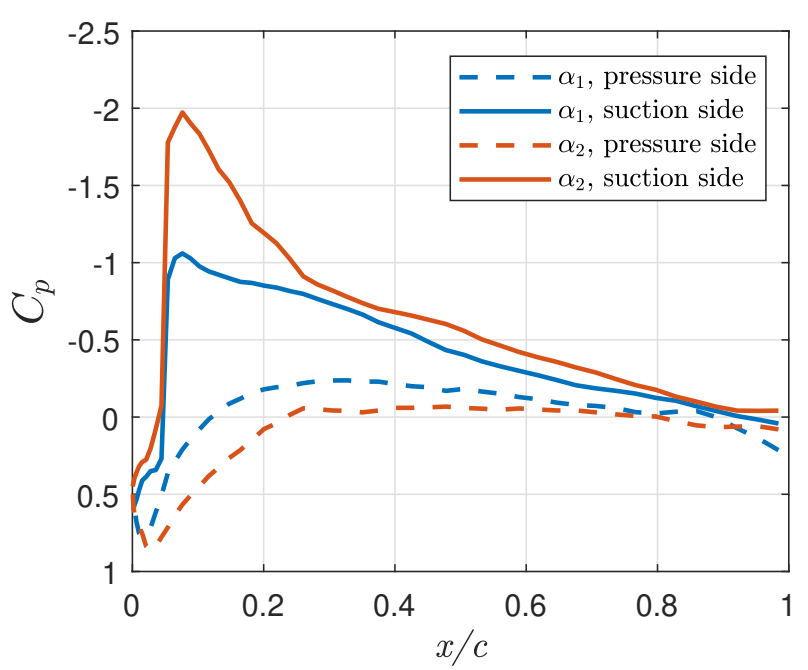

(b) $z / s=0.4$

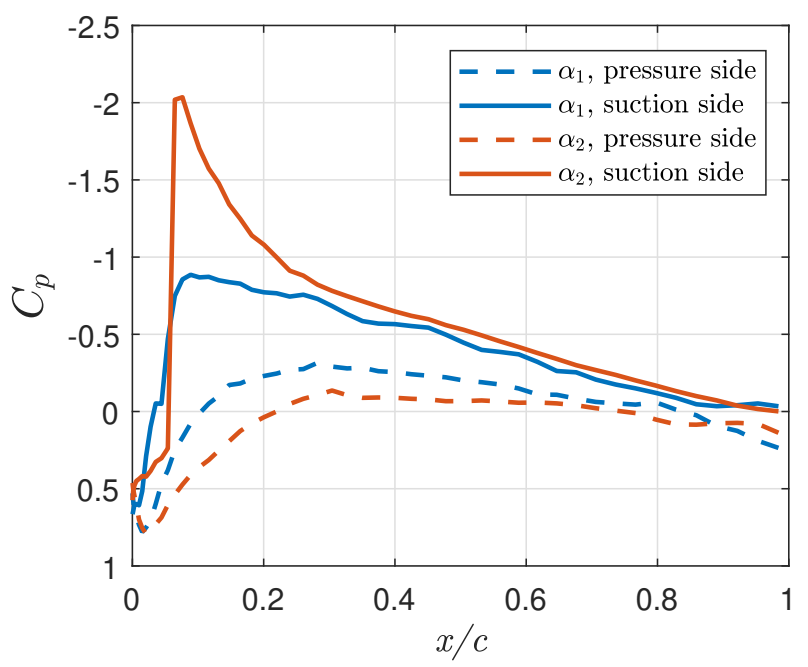

(d) $z / s=0.8$

Figure 9: LPT-based surface pressure distribution at four different spanwise locations

The lift distributions obtained by integrating the pressure difference along the chord, as well as from the circulation-based method are compared in Fig. 10. The lift distributions from both LPT-based methods show a plateau of the lift along most of the span and a drop towards the wing root and tip. This behavior is expected from aerodynamics theory due to the presence of the wing fixture at the root and the tip vortex. The pressure-based lift is consistently lower than the circulation-based lift, which is likely due to the reduction of the suction due to experimental errors near the leading edge, which were observed in Fig. 9. Furthermore, the pressure-based lift shows a larger level of random variation along the span compared to the circulationbased method, which is expected because the pressure-based method uses only LPT data measured in the vicinity of the wing, whereas, for the circulation-based method, several different integration paths were used and averaged.

A quantitative assessment of the accuracy of the LPT-based loads determination is performed by integrating the LPT-based lift result from the pressure- and circulation-based approaches along the span. These results are compared with the balance measurements in Tab. 1. The differences of the circulation-based method to the force balance measurements are considerably smaller than for the pressure-based method, by around $10 \%$. The difference to the force balance for both methods is increased for $\alpha_{2}$ compared to $\alpha_{1}$, but overall it remains below $5 \%$ for the circulation-based and below $15 \%$ for the pressure-based method. 


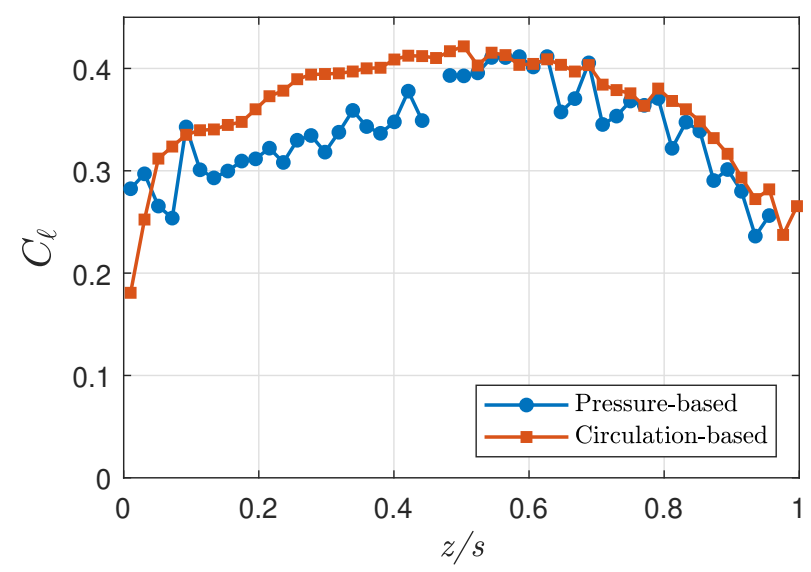

(a) $\alpha_{1}=5^{\circ}$

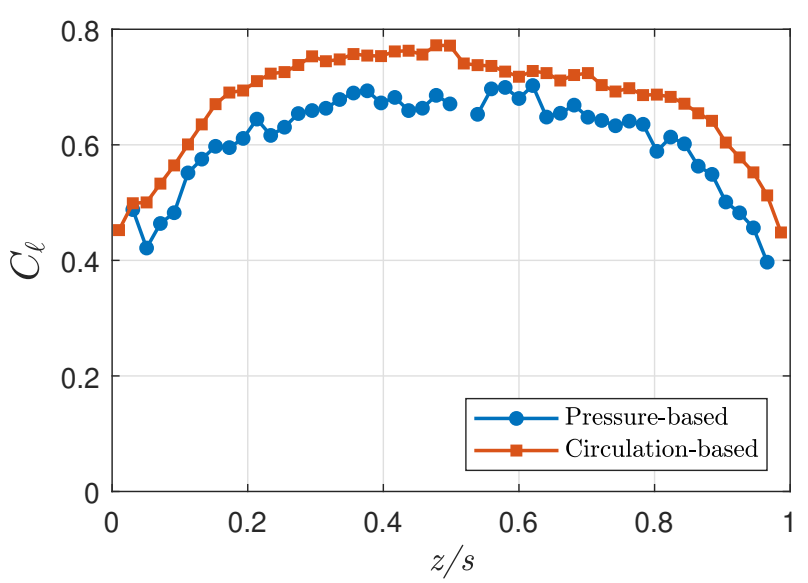

(b) $\alpha_{2}=10^{\circ}$

Figure 10: Comparison of the lift distribution across the span with different methods

Table 1: Comparison of the lift force from the LPT-based methods with the force balance measurements

\begin{tabular}{lrrrr}
\hline \hline \multirow{2}{*}{ Method } & \multicolumn{2}{c}{$\alpha_{1}=5^{\circ}$} & \multicolumn{2}{c}{$\alpha_{2}=10^{\circ}$} \\
& Lift force & Difference & Lift force & Difference \\
\hline Force balance & $4.12 \mathrm{~N}$ & & $7.83 \mathrm{~N}$ & \\
Pressure-based & $3.74 \mathrm{~N}$ & $-9.3 \%$ & $6.75 \mathrm{~N}$ & $-13.7 \%$ \\
Circulation-based & $4.00 \mathrm{~N}$ & $-2.9 \%$ & $7.48 \mathrm{~N}$ & $-4.5 \%$ \\
\hline \hline
\end{tabular}

\section{Conclusion}

This study has demonstrated the non-intrusive flowfield-based determination of aerodynamic loads on a flexible wing with a gridless approach based on discrete LPT measurement data. Contrary to most approaches in the published literature, the loads were not determined from ensemble-averaged data on a Cartesian grid, but instead by first determining the deformed shape of the wing from LPT measurements of structural markers and then analyzing the discrete LPT flowfield data in a local coordinate system, which is given by the measured wing shape. The aerodynamic loads were evaluated for two different angles of attack, $\alpha_{1}=5^{\circ}$ and $\alpha_{2}=10^{\circ}$ and with two different methods, which are the pressure-based and the circulation-based approaches. For the pressure-based approach, flow measurements in the immediate vicinity of the wing are necessary. The LPT data in this region is associated with a considerable amount of random error, and it is furthermore more challenging to analyze due to the presence of the viscous boundary layer. These challenges were addressed in this study by performing an extrapolation of the surface pressure along surface normals. However, the lift estimation based on a chordwise integration of the pressure difference was up to $13.7 \%$ lower than the reference lift measured with the force balance, mostly due to the erroneous surface pressure evaluation in the leading edge region of the wing. In contrast, the circulation-based approach does not require measurements in the near vicinity of the wing, as such it suffers to a lesser extent from the measurement-related issues. When comparing the integral lift force along the span from this approach with force balance measurements, a very good agreement is observed, with differences in the lift force of less than $5 \%$. Therefore, the circulation-based approach is preferred for a more accurate and robust lift determination, if the pressure distribution itself is of no direct interest. A topic for future research is a performance assessment of the gridless approach by comparison to results obtained with an ensemble-averaging approach. Additional topics include the improvement of the pressure extrapolation in the leading edge region and the application of the gridless approach to unsteady flows and flow situations in which the aerodynamic loads are more critically affected by viscous effects. 


\section{Acknowledgements}

The authors gratefully acknowledge the help of Adrián Grille Guerra with the Lagrangian particle tracking measurements during the wind tunnel experiments. This work has been carried out in the context of the HOMER (Holistic Optical Metrology for Aero-Elastic Research) project that has received funding from the European Union's Horizon 2020 research and innovation programme under grant agreement No. 769237.

\section{References}

Agüera N, Cafiero G, Astarita T, and Discetti S (2016) Ensemble 3D PTV for high resolution turbulent statistics. Measurement Science and Technology 27

Avin O, Raveh DE, Drachinsky A, Ben-Shmuel Y, and Tur M (2021) An experimental benchmark of a very flexible wing. in AIAA Scitech 2021 Forum. American Institute of Aeronautics and Astronautics

Dimitriadis G (2017) Introduction to Nonlinear Aeroelasticity. John Wiley \& Sons

Faleiros DE, Tuinstra M, Sciacchitano A, and Scarano F (2019) Generation and control of helium-filled soap bubbles for PIV. Experiments in Fluids 60

Jux C, Sciacchitano A, Schneiders JFG, and Scarano F (2018) Robotic volumetric PIV of a full-scale cyclist. Experiments in Fluids 59

Mertens C, Sciacchitano A, van Oudheusden BW, and Sodja J (2021) An integrated measurement approach for the determination of the aerodynamic loads and structural motion for unsteady airfoils. Journal of Fluids and Structures 103

Mitrotta FMA, Sciacchitano A, Sodja J, de Breuker R, and van Oudheusden BW () Experimental investigation of the fluid-structure interaction between a flexible plate and a periodic gust by means of robotic volumetric PIV. in CJ Kähler, R Hain, S Scharnowski, and T Fuchs, editors, 13th International Symposium on Particle Image Velocimetry. pages 645-656

Olasek K and Karczewski M (2021) Velocity data-based determination of airfoil characteristics with circulation and fluid momentum change methods, including a control surface size independence test. Experiments in Fluids 62

Rival DE and van Oudheusden BW (2017) Load-estimation techniques for unsteady incompressible flows. Experiments in Fluids 58

Scarano F, Ghaemi S, Caridi GCA, Bosbach J, Dierksheide U, and Sciacchitano A (2015) On the use of helium-filled soap bubbles for large-scale tomographic PIV in wind tunnel experiments. Experiments in Fluids 56

Schanz D, Gesemann S, and Schröder A (2016) Shake-the-box: Lagrangian particle tracking at high particle image densities. Experiments in Fluids 57

Schanz D, Gesemann S, Schröder A, Wieneke B, and Novara M (2013) Non-uniform optical transfer functions in particle imaging: calibration and application to tomographic reconstruction. Measurement Science and Technology 24

Schlichting H and Gersten K (2017) Boundary-Layer Theory. Springer. 9th edition

Sciacchitano A and Scarano F (2014) Elimination of PIV light reflections via a temporal high pass filter. Measurement Science and Technology 25

Sharma SD and Deshpande PJ (2012) Kutta-Joukowsky theorem in viscous and unsteady flow. Experiments in Fluids 52:1581-1591

van Oudheusden BW (2013) PIV-based pressure measurement. Measurement Science and Technology 24

Wieneke B (2008) Volume self-calibration for 3D particle image velocimetry. Experiments in Fluids 45:549_ 556 